\title{
CONCEPÇÃO DE UM SISTEMA DE VISUALIZAÇÃO DE INFORMAÇÕES GEOGRÁFICAS VIA WEB SOBRE A DESERTIFICAÇÃO
}

\author{
SANTOS, Saulo Medrado dos - saulomedrado1@gmail.com \\ Universidade Federal da Bahia / UFBA
}

LEAL, Brauliro Gonçalves - brauliro.leal@univasf.edu.br Universidade Federal do Vale do São Francisco / UNIVASF

GODOY NETO, Mario - mario.godoy@univasf.edu.br Universidade Federal do Vale do São Francisco / UNIVASF

TAURA, Tatiana Ayako - tatiana.taura@embrapa.br Empresa Brasileira de Pesquisa Agropecuária / EMBRAPA

\begin{abstract}
RESUMO: Com o avanço do acesso e disseminação de informações via web, a divulgação e o acompanhamento da evolução da desertificação através de um Sistema de Visualização de Informações Geográficas via Web torna-se uma ferramenta útil para gestão das áreas em processo de desertificação, como subsidio a elaboração de planos de revitalização dentre outras aplicabilidades. Nessa perspectiva, este trabalho teve por objetivo a construção de um sistema de visualização via web em torno da temática desertificação. Utilizando-se de metodologia ainda pouco discutida nas revistas científicas. Buscando dar publicidade aos dados pertencentes aos centros de pesquisa, como também a dados climáticos gerados para região escolhida como recorte de estudo. Tendo as etapas metodológicas de estruturação do sistema base da aplicação; armazenamento da base de dados; criação e edição das classes dos atributos para os dados geoespaciais e a construção do servidor de mapas o qual foi denominado "DesertGIS". A aplicação desenvolvida neste trabalho mostrou-se eficiente para a visualização e disponibilização de mapas interativos com diversas funcionalidades para visualização, interação e exportação das informações geográficas de interesse.
\end{abstract}

PALAVRAS-CHAVES: mapserver; i3geo; WEBGIS; desertificação.

CONCEPCIÓN DE UN SISTEMA DE VISUALIZACIÓN DE INFORMACIÓN GEOGRÁFICA VÍA WEB SOBRE LA DESERTIFICACIÓN

RESUMEN: Con el avance del acceso y diseminación de informaciones vía web, la divulgación y el seguimiento de la evolución de la desertificación a través de un Sistema de Visualización de Informaciones Geográficas vía Web se convierte en una herramienta útil para la gestión de las áreas en proceso de desertificación, como subsidio a la elaboración de planes de revitalización entre otras aplicaciones. En esta perspectiva, este trabajo tuvo por objetivo la construcción de un sistema de visualización vía web en torno a la temática desertificación. Utilizándose de metodología aún poco discutida en las revistas científicas. Buscando dar publicidad a los datos pertenecientes a los centros de investigación, así como a datos climáticos generados para región elegida como recorte de estudio. Teniendo las etapas metodológicas de estructuración del sistema base de la aplicación; almacenamiento de la base de datos; creación y edición de las clases de atributos para los datos geoespaciales y la construcción del servidor de mapas que se denominó "DesertGIS". La aplicación desarrollada en este trabajo se mostró eficiente para la visualización y disponibilidad de mapas interactivos con diversas funcionalidades para visualización, interacción y exportación de las informaciones geográficas de interés.

PALABRAS CLAVE: mapserver; i3geo; WEBGIS; desertificación.

CONCEPTION OF A VISUALIZATION SYSTEM FROM GEOGRAPHICAL INFORMATION VIA THE WEB ON DESERTIFICATION 


\begin{abstract}
With the advancement of access and dissemination of information via the web, the dissemination and monitoring of the evolution of desertification through a Geographic Information Visualization System via the Web becomes a useful tool for the management of areas in the desertification process, such as elaboration of revitalization plans among other applications. From this perspective, this work had the objective of constructing a web visualization system around the desertification theme. Using a methodology not yet discussed in scientific journals. In order to publicize the data belonging to the research centers, as well as the climatic data generated for the chosen region as a study clipping. Having the methodological steps of structuring the base system of the application; storage of the database; creating and editing the attributes classes for the geospatial data and building the map server which was called "DesertGIS". The application developed in this work proved to be efficient for the visualization and provision of interactive maps with several functionalities for visualization, interaction and export of the geographic information of interest.
\end{abstract}

KEYWORDS: mapserver; i3geo; WEBGIS; desertification.

CONCEPTION D'UN SYSTĖME DE VISUALISATION D'INFORMATIONS GÉOGRAPHIQUES VIA LE WEB ON DESERTIFICATION

RESUME: Avec l'avancement de l'accès et la diffusion de la diffusion de l'information sur le Web et le suivi de l'évolution de la désertification à travers une Vue d'information géographique System Web devient un outil utile pour les domaines de la gestion dans le processus de désertification, en tant que subvention préparation de plans de revitalisation entre autres domaines d'application. Dans cette perspective, cette étude visait à construire un système de visualisation basé sur le Web autour de la désertification à thème. En utilisant la méthodologie n'est pas abordée dans des revues scientifiques. Cherchant à la diffusion des données appartenant à des centres de recherche, ainsi que les données climatiques générées pour la région choisie comme l'écrêtage de l'étude. Avoir les étapes méthodologiques de la structuration de la mise en œuvre du système de base; le stockage de la base de données; la création et la modification de classes d'attributs pour les données géospatiales et la construction du serveur de carte qui a été appelé "DesertGIS". L'application développée dans ce travail est avéré être efficace pour la visualisation et la livraison de cartes interactives avec de nombreuses fonctionnalités pour la visualisation, l'interaction et l'exportation de l'information géographique d'intérêt.

MOT CLÉS: mapserver; i3geo; WEBGIS; désertification.

\title{
INTRODUÇÃO
}

Estudos sobre os efeitos, localização e ações de combate em áreas que sofrem com o processo de desertificação são amplamente discutidos na literatura. Embora publicados, estas informações são de difícil acesso ao público não especializado. Os resultados e discussões acerca do tema ficam restritos a algumas instituições públicas ou privadas e poucos esforços são vistos quanto a possibilidade de disponibilizá-los para a comunidade não científica.

Dentre estas tecnologias, pode-se citar os Sistemas de Visualização de Informações Geográficas via Web que em conjunto com o Banco de Dados Geográfico (BDG) formam um conjunto de ferramentas de interação para a difusão de informações; tornando-se uma importante instrumento de controle sobre a evolução da desertificação, contribuindo para a identificação de áreas desertificadas podendo servir também como subsidio à elaboração de planos de revitalização visando a redução de áreas que sofrem com o processo de desertificação.

Sousa Neto (2009) aponta que através desse conjunto de ferramentas, os usuários ou clientes podem acessar as informações no formato original e 
realizar consultas em diferentes níveis de complexidades, gerando mapas interativos a partir de um Banco de Dados Geográfico (BDG)

Observa-se nos dias atuais o aumento expressivo na disponibilização da informação via Internet, de modo a facilitar o acesso aos mais diversos tipos de dados (MOREIRA, 2010). O emprego da web como um meio de utilização dos sistemas de visualização pode ser considerado um grande avanço no geoprocessamento e abre novas oportunidades, como os mapas em tempo real, divulgações a custos reduzidos, atualizações mais frequentes e com baixo custo, utilização de softwares livres, elaboração de conteúdo para sistemas personalizados, diversidade de fontes de dados e compartilhamento de informação geográfica.

Nessa perspectiva, este trabalho teve por objetivo a construção de um sistema de visualização via web em torno da temática desertificação. Buscando dar publicidade aos dados pertencentes aos centros de pesquisa, como também a dados climáticos gerados para região escolhida como recorte de estudo.

As ferramentas utilizadas como base para criação do sistema proposto foi o Mapserver software ${ }^{1}$ principal sob o qual foi desenvolvido a interface i3Geo ${ }^{2}$. As principais características que levaram a escolha do Mapserver em conjunto com o I3geo como servidor de mapas para o referido trabalho foram baseadas no fato desta ferramenta não se limitar ao fornecimento de mapas interativos, oferecendo um conjunto de aplicativos que atendem às necessidades de construção da Infraestrutura Nacional de Dados Espaciais (INDE ${ }^{3}$ ).

Além de ser compatível com as especificações difundidas pela Open Geospatial Consortium (OGC ${ }^{4}$ ) como WMS (Web Map Service) permitem a leitura de múltiplas camadas contendo vetores e/ou imagens; WFS (Web Feature Service) apresenta uma forma de acesso (inserção, atualização, exclusão e análise) à feição através do ambiente WEB (HTTP) e WCS (Web Coverage Service) fornece comunicação eletrônica baseada na arquitetura cliente/servidor de dados geográficos.

A arquitetura responsável pelo processamento das informações, está dividido em módulos ou processos distintos. Sendo um processo responsável pela manutenção da informação (BDG e servidores) e outro responsável pela obtenção dos dados (os clientes). Possibilitando pelo lado servidor, o acesso e a integridade de dados; pelo lado cliente, disponibilização de ambiente individual de interatividade com recursos gráficos.

\footnotetext{
${ }^{1} \mathrm{O}$ Mapserver é um agregado de ferramentas e recursos do tipo código aberto desenvolvido pela Universidade de Minesota e pela NASA (National Aeronautics and Space Administration) em 1996 (PARMA, 2007; MAPSERVER, 2012).

2 A sigla "i3Geo" significa "Interface Integrada para Internet de Ferramentas de Geoprocessamento". Trata-se de um software livre, licenciado como GPL (Licença Pública Geral) e criado pelo Ministério do Meio Ambiente (MMA) do Brasil em 2004 (I3GEO, 2012).

${ }^{3}$ No Brasil, a IDE nacional, ou INDE, está a cargo da Comissão Nacional de Cartografia (CONCAR) e utiliza a seguinte definição: "conjunto integrado de tecnologias; políticas; mecanismos e procedimentos de coordenação e monitoramento; padrões e acordos, necessário para facilitar e ordenar a geração, o armazenamento, o acesso, o compartilhamento, a disseminação e o uso dos dados geoespaciais de origem federal, estadual, distrital e municipal".

${ }^{4} \mathrm{O}$ Open Geospatial Consortium, OGC, é uma organização voluntária internacional que desenvolve e implementa padrões para conteúdos e serviços geoespaciais na web.
} 


\section{MATERIAIS E MÉTODOS}

A área de estudo abrange os municípios de Juazeiro, Casa Nova, Sobradinho e Curaçá no norte da Bahia. Localizada entre as coordenadas $10^{\circ} 03^{\prime} 23.01^{\prime \prime}$ e $-8^{\circ} 28^{\prime} 28.10^{\prime \prime}$ de latitude e $-41^{\circ} 56^{\prime} 00.36^{\prime \prime}$ e -39¹5'22.19" de longitude (Figura 1).

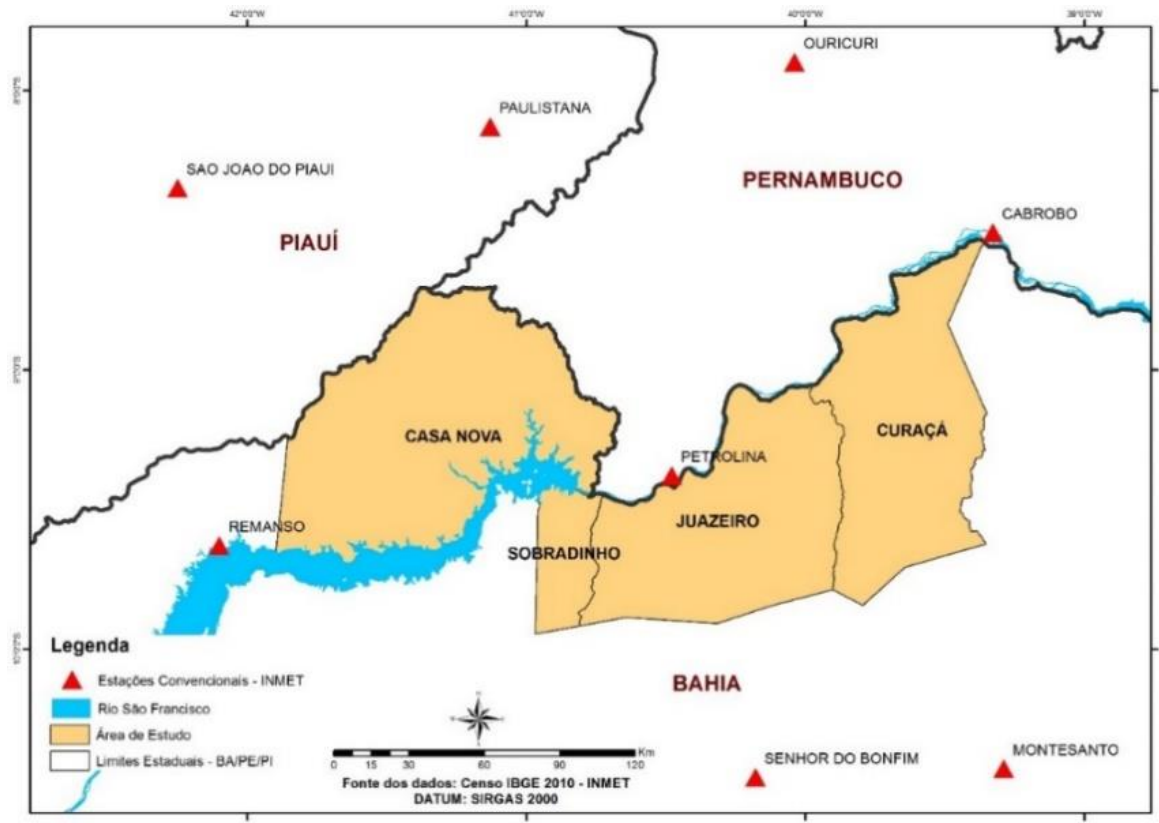

Figura 1 - Localização da área de estudo. Fonte: Os autores

A proposta do sistema concebido, inicialmente, para disponibilização de dados sobre a desertificação para área de estudo segue o conceito cliente/servidor, caracteriza-se pelo envio de requisições de dados pelo usuário (cliente) para algum dos servidores conectados a uma rede de computadores, para o trabalho o servidor utilizado foi o Mapserver em conjunto com o I3geo, o qual pode aceitar tais requisições, processá-las e retornar o resultado para o cliente (Figura 2). 


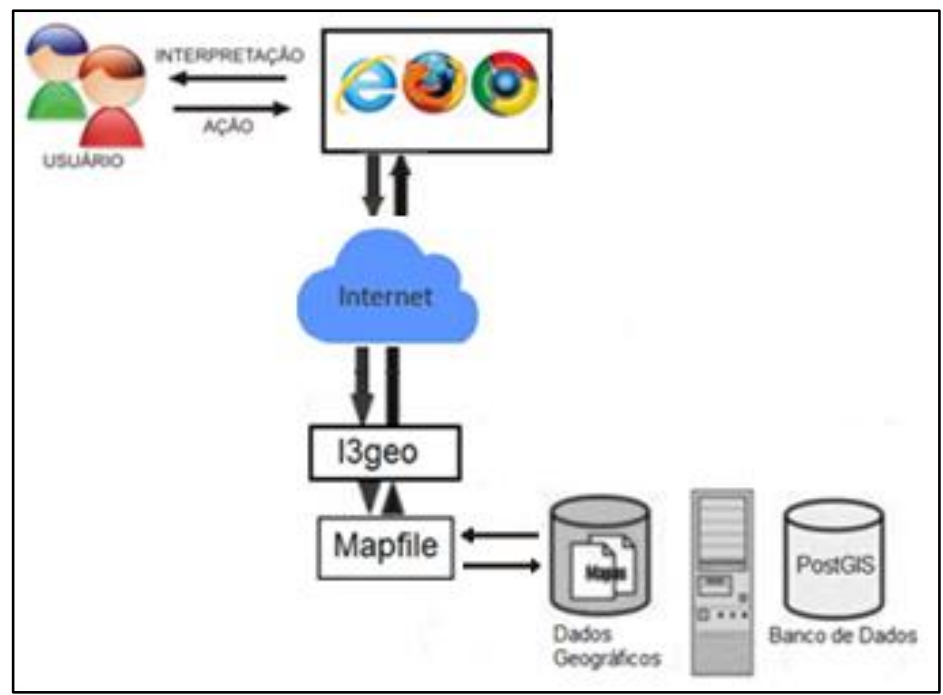

Figura 2 - Visualização da proposta do sistema de visualização de informações geográficas via web. Fonte: Os autores

Tendo em conta os custos significativos que o desenvolvimento de um sistema de visualização via web pode assumir, optou-se pela utilização de softwares livres na elaboração do mesmo. Os softwares utilizandos foram: Apache, versão 2.2.22; Mapserver CGI, versão 6.0.3; PHP, versão 5.4.3; I3Geo, versão 6.0 e o SGBD PostgreSQL, versão 9.4 .3 com a extensão espacial PostGIS, versão 2.1. A estrutura do projeto segue a representada pelo diagrama a seguir (Figura 3):

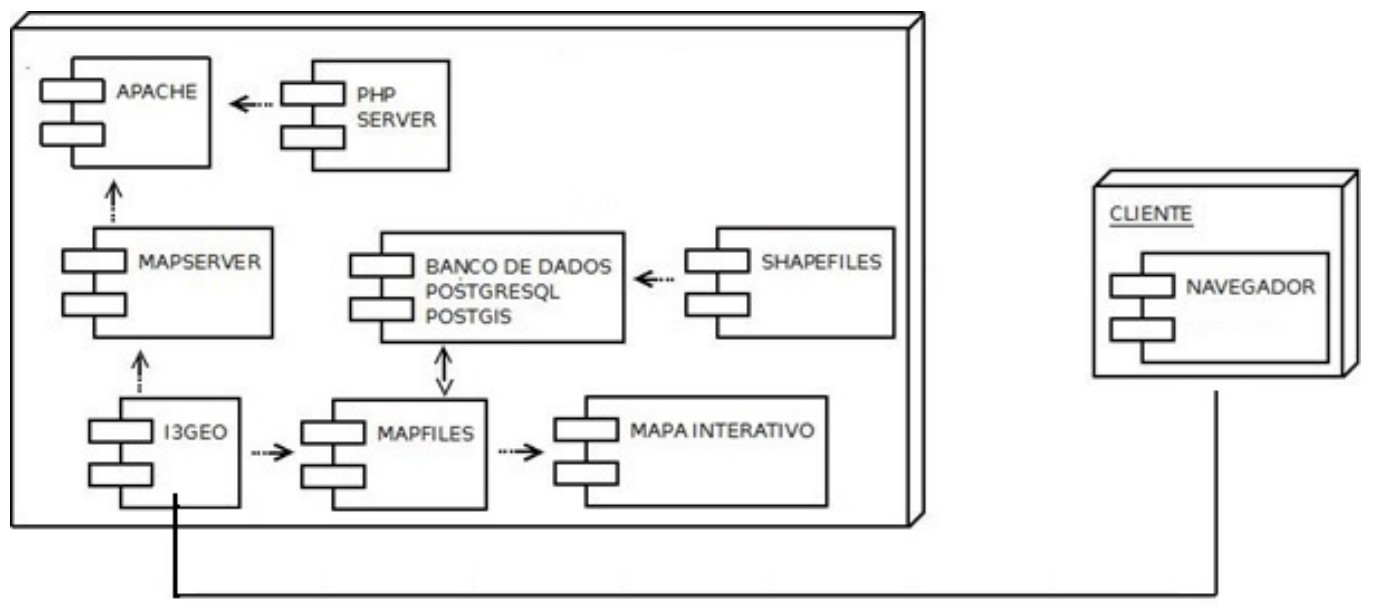

Figura 3 - Diagrama de componentes para o sistema. Fonte: Os autores

A partir da Figura 3 importante destaque deve se dar aos seguintes componentes:

- Shapefiles 5 :

\footnotetext{
${ }^{5}$ Formato de arquivo desenvolvido e regulamentado pela ESRI contendo dados geospaciais em forma de vetor. Descrevem geometrias: pontos, linhas e polígonos.
} 
Os dados geográficos em formato shapefile obtidos e trabalhados para auxiliar na localização e contextualização das análises dos dados foram: cobertura vegetal; uso do solo; classes de solo; vulnerabilidade do solo à desertificação; geomorfologia; rede hidrográfica; limites e localidades municipais; localização das estações meteorológicas da área de abrangência assim como dados de precipitação, temperatura, evapotranspiração, índice de aridez e suscetibilidade à desertificação para região.

Os dados vetoriais de Vegetação, Solo, Uso do Solo; Vulnerabilidade do Solo à Desertificação, Geomorfologia e Rede Hidrográfica foram obtidos junto ao Laboratório de Geoprocessamento e Sensoriamento Remoto da Embrapa Semiárido.

Os dados de Limites e Localidades Municipais foram obtidos através do IBGE$^{6}$ também em formato vetorial (shapefile).

Os dados climáticos foram obtidos através do INMET $^{7}$ e transformados em formato vetorial (shapefile) pelos autores.

Nota-se que a base cartográfica não foi elaborada por um processo contínuo de levantamento e tratamento de dados, mas formada por um conjunto de informações já existentes obtidos de maneiras diversas por diferentes órgãos.

Seguindo as recomendações atuais para padronização do dado cartográfico, utilizou-se o sistema de referência oficial em uso atualmente no Brasil, SIRGAS 2000 (IBGE, 2005).

- Banco de Dados Geográfico (BDG):

Para a organização e armazenamento dos dados da etapa anterior utilizou-se como ferramenta de banco de dados principal o PostgreSQL, versão 9.4.3 em conjunto com sua extensão espacial PostGIS, versão 2.1.

- Mapfile:

A fase de construção e edição dos Mapfiles é uma fase crítica no processo de preparação dos mapas interativos. São eles que definem as características de cada tema disponível para leitura pelo servidor de mapas.

A edição das cores para os atributos contidos nos dados geográficos obedeceu ao indicado nos Manuais Técnicos em Geociências para Vegetação (IBGE, 2012) e Pedologia (IBGE, 2007). Posteriormente foram incluídos na pasta "i3geo/temas".

- Servidor de mapas:

Utilizou-se o servidor de mapas Mapserver em sua versão 6.0.3 em conjunto com o I3Geo, em sua versão 6.0.

Foi instalado o ambiente onde o i3Geo funcionaria, o que significou configurar no computador um servidor WEB. Esse servidor necessita basicamente dos softwares Apache, PHP e Mapserver. É disponibilizado pelo pacote extensão MS4W (Mapserver for windows). Uma vez configurado 0

\footnotetext{
${ }^{6} \mathrm{ftp}: / /$ geoftp.ibge.gov.br/../../malhas_digitais/

${ }^{7}$ http://www.inmet.gov.br/projetos/rede/pesquisa/
} 
servidor, foi copiada a pasta contendo os arquivos do i3Geo, para a pasta de

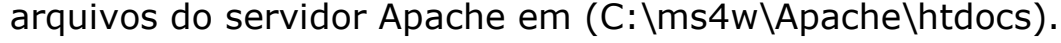

O acesso ao sistema de administração responsável pela manutenção do catálogo de temas, criação e edição dos arquivos Mapfiles é feita através da URL: http://localhost/i3geo/admin. O acesso pelo cliente para visualização dos mapas temático no I3geo é feito através da URL: http://localhost/i3geo/interface/black_ol.htm

A manutenção do catálogo de temas é a principal tarefa dos administradores. Manter o catálogo significa configurar as camadas que poderão ser utilizadas nos mapas interativos, além de organizá-las para tornar sua localização mais fácil e intuitiva. Essa organização é feita construindo-se uma "Árvore de Temas", composta por menus, grupos, subgrupos e temas em uma estrutura hierárquica.

- Cliente:

Realiza a navegação no mapa interativo e operações através do navegador pelo link: http://localhost/i3geo/interface/black_ol.htm. Na Tabela 1 é mostrado alguns exemplos de ferramentas/funcionalidades que o cliente utiliza para operação no mapa interativo.

Tabela 1 - Ferramentas de navegação e análise para o cliente

\begin{tabular}{|c|c|c|c|}
\hline Ferramenta & Funcionalidade & Ferramenta & Funcionalidade \\
\hline & $\begin{array}{l}\text { Altera a escala do mapa } \\
\text { ajustando-a para } \\
\text { mostrar a mesma } \\
\text { abrangência geográfica } \\
\text { da inicialização. }\end{array}$ & & $\begin{array}{l}\text { Amplia o mapa - desloca o } \\
\text { ponto clicado para centro da } \\
\text { tela ou amplia a região } \\
\text { indicada por um retângulo. }\end{array}$ \\
\hline & $\begin{array}{l}\text { Desloca a região visível } \\
\text { no mapa. }\end{array}$ & & $\begin{array}{l}\text { Mostra informações sobre um } \\
\text { ponto no mapa. }\end{array}$ \\
\hline & $\begin{array}{l}\text { Mostra informações } \\
\text { resumidas sobre um } \\
\text { ponto clicado no mapa. } \\
\text { Basta clicar no mapa a } \\
\text { qualquer tempo. }\end{array}$ & & $\begin{array}{l}\text { Mostra a extensão geográfica } \\
\text { atual em coordenadas } \\
\text { geográficas. }\end{array}$ \\
\hline & $\begin{array}{l}\text { Abre/fecha o mapa de } \\
\text { referência. }\end{array}$ & & Legenda \\
\hline & $\begin{array}{l}\text { Mostra dados } \\
\text { Meteorológicos. }\end{array}$ & & Abre lente de ampliação. \\
\hline & $\begin{array}{l}\text { Abre o Google Maps, } \\
\text { mostrando uma imagem } \\
\text { de satélite da região } \\
\text { vista no mapa principal. }\end{array}$ & & $\begin{array}{l}\text { Abre as ferramentas para } \\
\text { seleção de elementos de um } \\
\text { tema. Os elementos } \\
\text { selecionados podem ser } \\
\text { utilizados em outras } \\
\text { operações, como buffer e } \\
\text { seleção por tema. }\end{array}$ \\
\hline
\end{tabular}




\begin{tabular}{lll}
$\begin{array}{l}\text { Redesenha o mapa com } \\
\text { as configurações } \\
\text { iniciais. }\end{array}$ & $\begin{array}{l}\text { Mede a distância entre dois ou } \\
\text { mais pontos clicados no mapa } \\
\text { (menor distância). }\end{array}$ \\
\hline $\begin{array}{l}\text { Mede a área de um } \\
\text { polígono desenhado na } \\
\text { tela. }\end{array}$ & $\begin{array}{l}\text { Insere pontos no mapa em } \\
\text { coordenadas geográficas. Os } \\
\text { pontos incluídos podem ser } \\
\text { transformados em linhas ou } \\
\text { polígonos. }\end{array}$ \\
\hline
\end{tabular}

Fonte: Os autores

\section{RESULTADOS E DISCUSSÕES}

A partir dos métodos descritos, foi implementado um sistema de visualização via web com informações relativas à desertificação para proporcionar aos usuários interessados uma combinação de informações de mapas de solo; cobertura e uso do solo; geomorfologia; rede hidrográfica; juntamente com dados climáticos com a finalidade de embasar os possíveis usuários para tomada de decisão. Foi dado ao produto gerado o nome de "DesertGIS".

\section{IMPORTAÇÃO DOS ARQUIVOS PARA O BDG - POSTGRESQL/POSTGIS}

Como resultado da importação dos dados ao BDG foi possível realizar algumas consultas geográficas a fim de construir as tabelas que serviram de base para a visualização dos mapas temáticos a serem reproduzidos no "DesertGIS".

A Figura 4 representa o diagrama de relacionamento entre a Tabela "spatial_ref_sys" principal entidade (chave principal) do banco de dados e as tabelas geradas a partir da importação ao BDG "desertgis". As quais se relacionam com a primeira e são independentes entre si. 


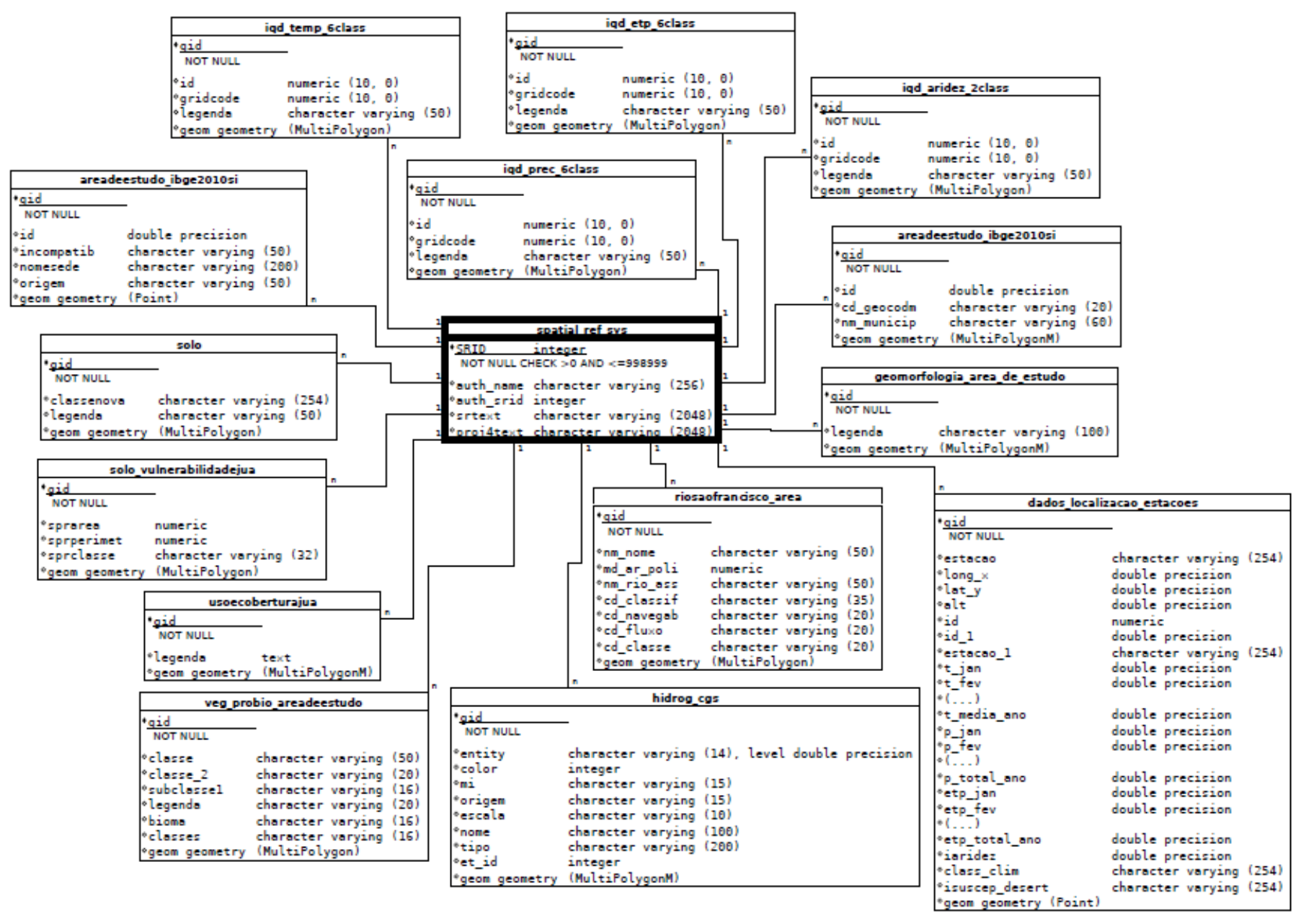

Figura 4 - Diagrama relacional das tabelas geradas no banco de dados "desertgis". Fonte: Os autores 
Com os resultados obtidos da importação, os arquivos geoespaciais (shapefiles) e demais informações elaboradas pelos autores para a realização do presente estudo, integraram o BDG mantendo-se todas as características originais dos dados, estando aptos para serem construídos os mapfiles.

\section{CONFIGURAÇÃO DOS ARQUIVOS .MAP (MAPFILES)} Figura 5.

A estrutura inicial para todos os arquivos criados segue o exposto na

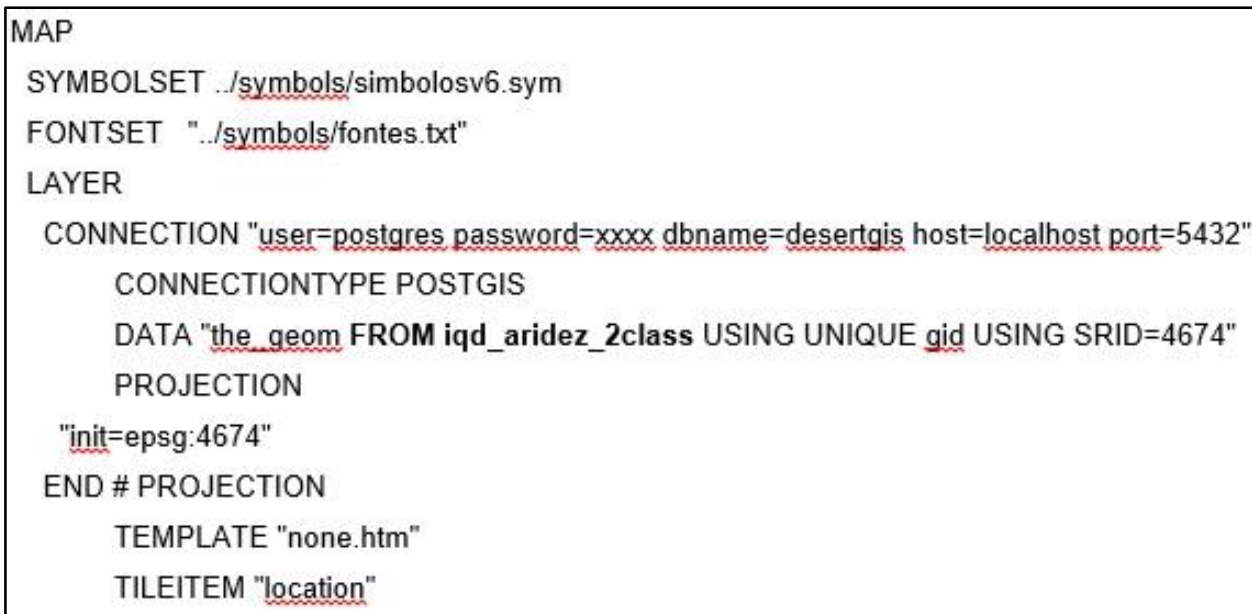

Figura 5 - Estrutura inicial para todos os mapfiles criados. Fonte: Os autores

Os comandos editados para a inclusão das fontes de informações ao sistema foram:

- "FROM" em destaque, que diz respeito ao nome do arquivo (shapefile) importado ao BDG;

- "CONNECTION", realiza a conexão entre o sistema e o BDG, ferramenta de armazenamento central deste estudo;

- "SRID" responsável pelo sistema de projeção (DATUM) do arquivo a ser trabalhado.

Na sequência é mostrada o resultado da edição do arquivo mapfile para o tema "IQD Índice de Aridez" (Figuras 6 e 7). 


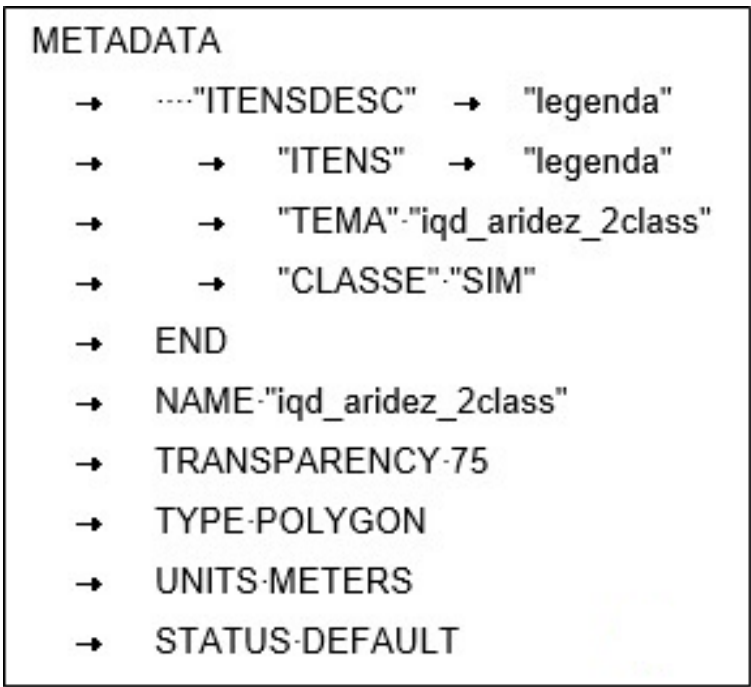

Figura 6 - Estrutura do mapfile para o tema criado - "IQD Índice de Aridez". Fonte: Os autores

Onde os comandos "ITENSDESC" e "ITENS" correspondem à coluna de atributos da Tabela; "TEMA" identificador do arquivo vetorial importado ao BDG; "NAME" identificação fornecida pelo administrador que poderá ser vista pelos clientes; "TYPE" forma geométrica e "UNITS" unidade métrica do arquivo vetorial importado ao BDG.

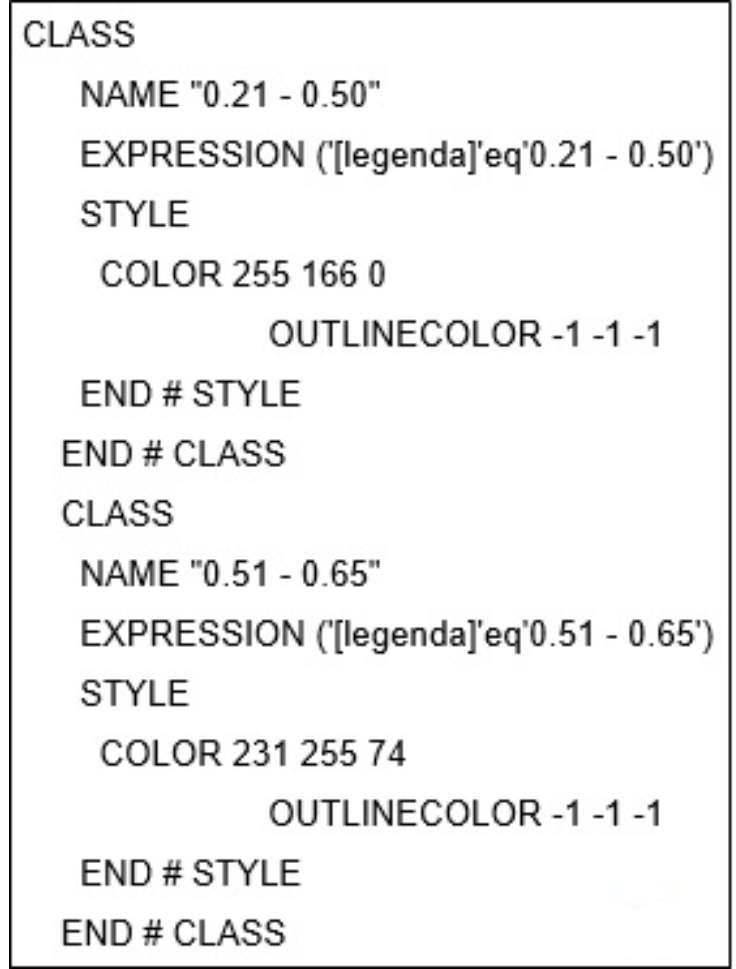

Figura 7 - Atribuição de cores ao mapfile para o tema criado - "IQD Índice de Aridez". Fonte: Os autores 
Os comandos "NAME" e "EXPRESSION" obedecem às classes da coluna de atributos dos arquivos (shapefile) importados e "COLOR" para inclusão das cores (RGB) que serão visualizadas na aba "Legenda" no sistema.

\section{MAPA INTERATIVO - MAPSERVER/I3GEO}

Quando o cliente acessa o link do projeto através do navegador, é enviada uma requisição para o MapServer, a qual é recebida pelo Apache. Este envia os arquivos (códigos das páginas e as imagens) para o cliente através da aplicação i3geo. Ao se visualizar um mapa com as informações requeridas e a cada alteração feita nos parâmetros da informação desejada uma nova requisição é enviada ao servidor atualizando, desta forma, o mapa. A visualização do "DesertGIS", produto desse estudo, pode ser visualizado através da Figura 8.

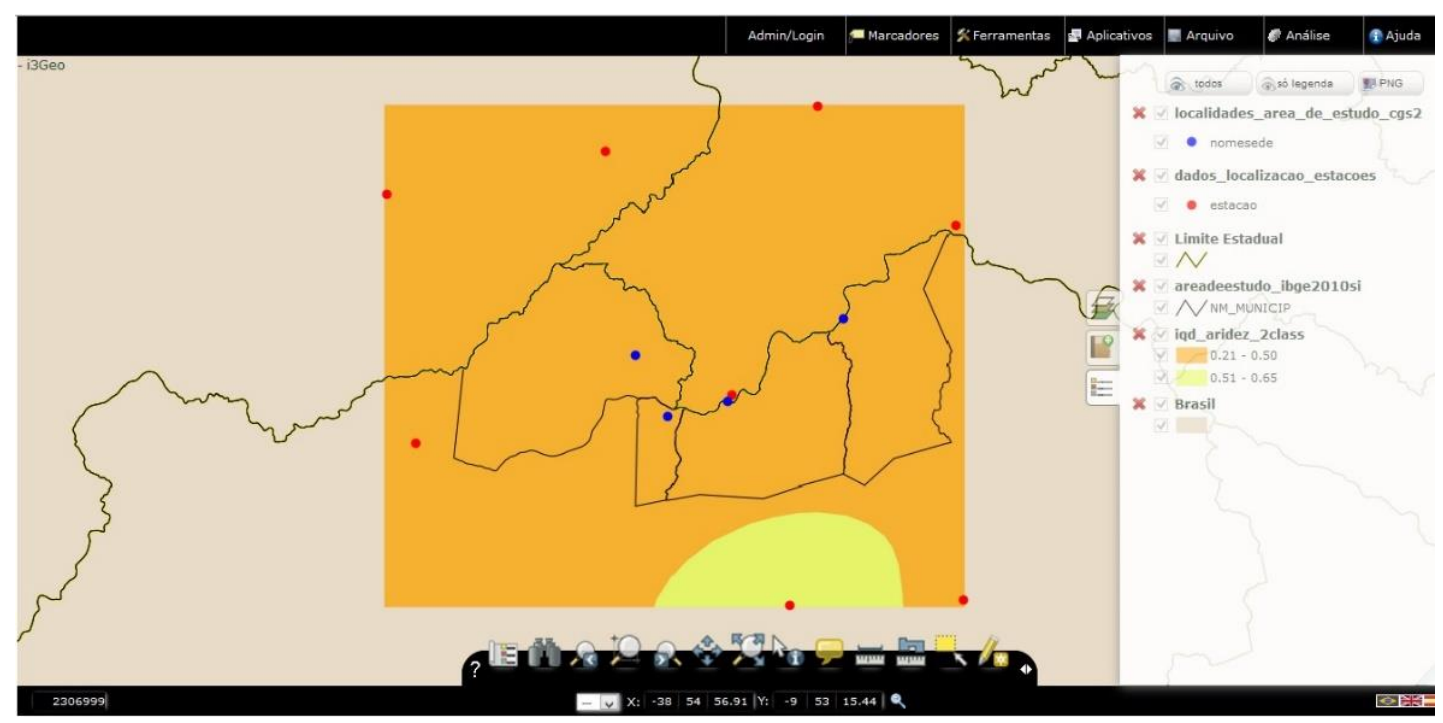

Figura 8 - Visualização do "DesetGIS" com os atributos "Localidades"; "Localização das Estações"; "Limites Municipais" e "Interpolação da Suscetibilidade à Desertificação". Fonte: Os autores

O acesso a base de dados é feito pela aba móvel localizada do lado direito da janela de navegação, na ferramenta chamada "Catálogo de Temas" (Figura 9). 


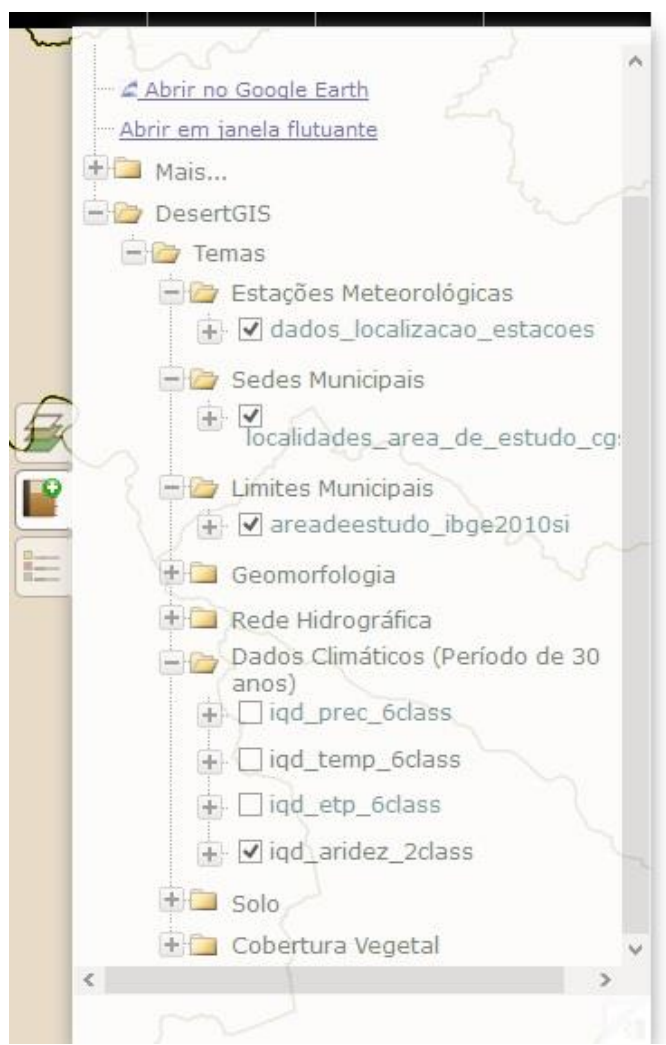

Figura 9 - Visualização da aba móvel "Catálogo de Temas". Fonte: Os autores

Uma vez selecionado o tema de interesse o mesmo é mostrado na tela principal do servidor. O "Catálogo de Temas" corresponde a configuração previamente realizada pelo administrador no item "Árvore de Temas" ferramenta responsável pela adição ou remoção dos temas no mapa interativo.

O resultado da criação da "Árvore de Temas" responsável pela organização das camadas do mapa temático é o que pode ser visto na Figura 10. 


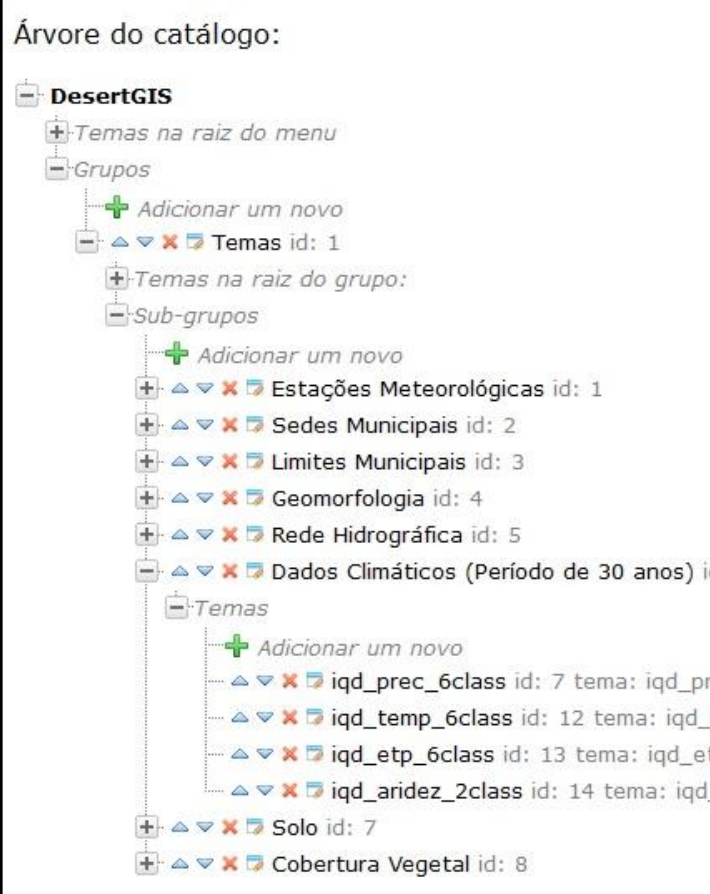

Figura 10 - Estrutura da "Arvore de temas" do "DesertGIS". Fonte: Os autores

Outra ferramenta utilizada pelo usuário para navegação no mapa interativo é a aba "Legenda" onde se encontra toda a simbologia classificada pelos administradores (Figura 11).

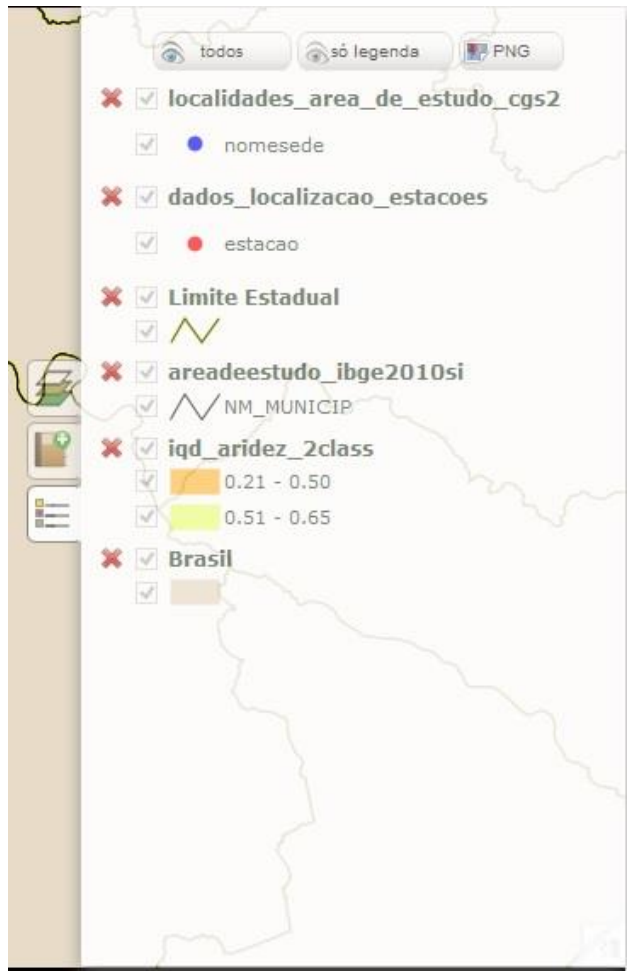

Figura 11 - Visualização da aba móvel "Legenda". Fonte: Os autores 
A aplicação desenvolvida neste trabalho, "DesertGIS", mostrou-se capaz de gerar visualização e disponibilizar mapas interativos de forma eficiente, mostrando ao usuário uma interface com diversas funcionalidades para interação, visualização e exportação das informações geográficas de interesse. Além disso, possibilita a sobreposição espacial simultânea de diversos mapas temáticos, permitindo analisar camadas de informação separadamente ou em conjunto.

\section{CONCLUSÕES}

De acordo com os resultados obtidos neste trabalho, concluiu-se que:

- A metodologia utilizada mostrou-se capaz de desenvolver um Sistema de Visualização via Web.

- Mostrou-se eficiente mesmo com número significativo de camadas para carregamento. Possibilita a sobreposição espacial simultânea de diversos mapas temáticos, permite analisar camadas de informação separadamente ou em conjunto

- Houve integridade para com os dados trabalhados não gerando perda ou inconsistência de dados.

- Toda simbologia de cores, linhas, extensão geográfica, das classes de atributos foi mantida.

- Os resultados mostram a possibilidade de utilização do sistema para as mais diversas temáticas não restringindo-se a região geográfica.

\section{REFERENCIAS BIBLIOGRAFICAS}

COSME, A. Projeto em sistemas de informação geográfica. Lisboa: Lidel, 2012.

I3GEO. I3geo 5.0 Manual do Administrador. 2012. 98 p. Disponível em: < http://www.i3geo.com.br/i3geo/documentacao/manual-admin-i3geo-6_0-

pt.pdf>. Acesso em: 15 abr. 2015.

MOREIRA, S. A. G. Cartografia multimídia: possibilidade para a produção de novos conhecimentos geográficos. In: Anais XXIV Congresso Brasileiro de Cartografia, 2010, Aracaju - SE

MAPSERVER. Open Source Web Mapping. MapServer 6.0.3 Documentation. 2012. 933 p. Disponível em: <http://www.mapserver.org>. Acesso em: 18 set. 2012.

PARMA, C. A. Mapas Cadastrais na Internet: Servidores de Mapas. In: XIII Simpósio Brasileiro de Sensoriamento Remoto (SBSR), 2007, Florianópolis, Anais... São José dos Campos: INPE, 2007. p. 1311-1319.

SOUSA NETO, W. P. Usando API do Google Maps para criar um mapa interativo: estudo de caso: Campus Viçosa. 2009. 42p. Monografia (Graduação em Engenharia de Agrimensura) - Universidade Federal de Viçosa, Viçosa, MG. 
VALIN, M. M. Uso de Softwares Livres para o Desenvolvimento de Sig Web de Acessibilidade a Atrativos Turísticos: Estudo de Caso da Cidade de Campinas-SP. 2009. 142 p. Dissertação (Faculdade de Engenharia Civil, Arquitetura e Urbanismo) - Universidade Estadual de Campinas, Campinas, SP. 